\title{
A CURRENT NEED FOR MODERN INSTRUMENTS IN FORENSIC PATHOLOGY: A WAY FORWARD
}

\author{
Paranitharan P. ${ }^{1}$, Hulathduwa S.R. ${ }^{2}$ \\ ${ }^{1}$ Department of Forensic Medicine, Faculty of Medicine, University of Kelaniya, \\ ${ }^{2}$ Senior Lecturer, Department of Forensic Medicine, University of Sri Jayewardenepura, \\ Sri Lanka
}

\begin{abstract}
Routine dissection of the cadaver followed by mandatory and ancillary investigations is still the most widely used method in forensic autopsy throughout the globe. In contrast to most other disciplines of medicine, instruments used in routine autopsy work remain plain, simple, unsophisticated and virtually unchanged for over a century. Incorporation of modern technology to improve these instruments to meet the new challenges such as minimizing the risk of biohazards, acquisition of precision in dissection, facilities for simultaneous imaging, sampling and archiving the information and finally making the dissection a less cumbersome a task with lesser degree of dependability on individual human skills has become the need of the day. The authors briefly discuss few initiative steps taken along this long pathway.
\end{abstract}

Key words: forensic pathology, modern instruments, advanced techniques, human cadaver.

Corresponding author: paran28@gmail.com

\section{INTRODUCTION}

Forensic Pathology is entering a new era. The credit mainly goes to few individuals who had established world class forensic centres in Canada, Australia, North America, England and Switzerland. However, standard instruments used in prosection of the human cadaver remain the same without much modification since the time known. Though great strides had been made to supplement the conventional autopsy by means of virtopsy; the requirement for the standard dissection of the cadaver to arrive at a cause of death and to address various other medico-legal issues still remain much the same. This phenomenon is more visible in the less developed parts of the world and this situation cannot be expected to be drastically changed in the near future.

\section{Purpose of this report}

The standard instruments currently used for dissection - the scalpel, scissors, brain knife and the saw can very well serve the required purposes. However, considering the astonishing advances of modern equipment, techniques and instruments in most other branches of medicine, the authors feel that a new array of modern instruments needs to be developed for routine autopsy purposes to address health and safety issues of the staff, achieve greater precision in dissection technique (including evisceration), minimize the hazels and cumbersomeness of the routine procedures and incorporate new technology in to the routine procedure such as digital imaging and storage. The main purpose of this article is to propose how the existing instruments could further be modified to achieve the above goals and to innovatively suggest how introduction of new instruments could take routine dissection procedure to greater heights. 


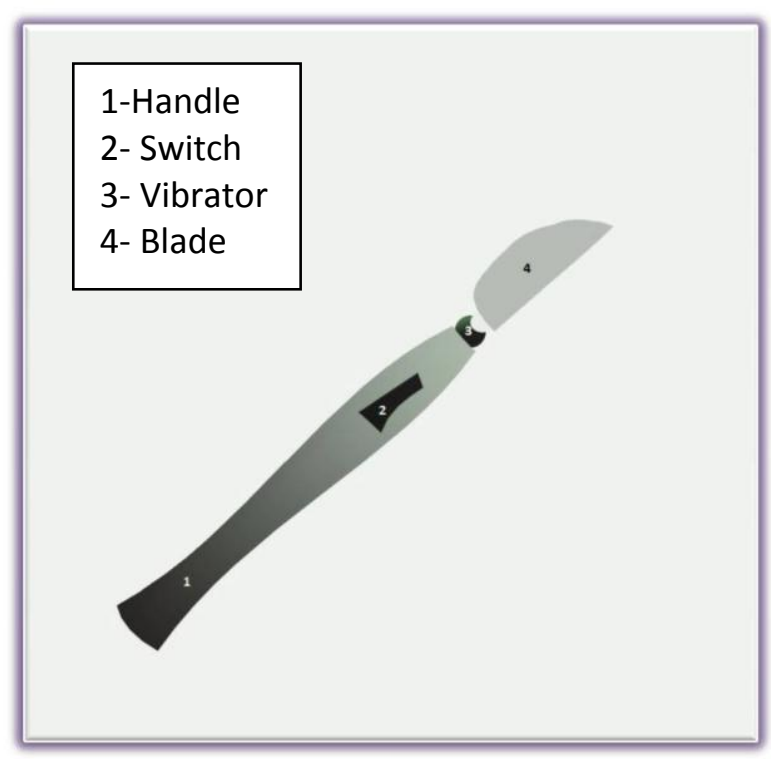

Figure 1: Electric Scalpel

As an example, the scalpel routinely used to dissect human cadaver, including sectioning of the calcified or non-calcified coronary arteries, could be modified into a battery operated one with incorporation of an electric motor between the handle and blade which would oscillate the blade to make the necessary dissections in a controlled manner. This simple modification would pave the way for a scalpel which is better designed and user friendly.

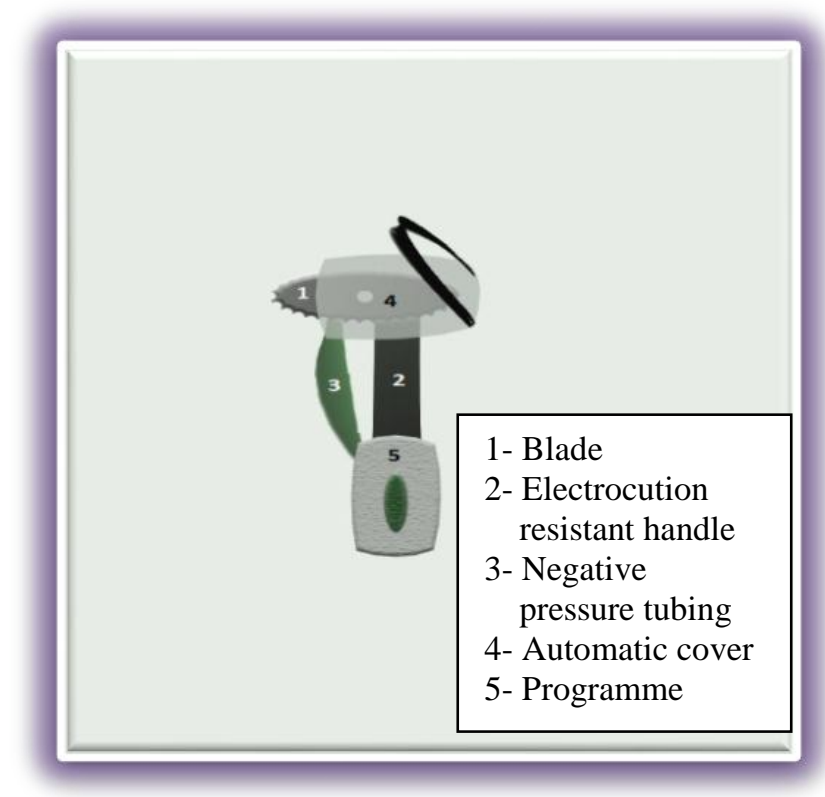

Figure 2: Modified Electric Saw
The electric saw, currently used for cutting the human skull to expose the brain, could be modified to a more user friendly, health and safety equipment specially designed for human skull removal and vertebral dissection. An installed program will be used to automatically determine the cutting depth of the blade when dissecting the human skull or vertebrae to prevent damage to the brain or the spinal cord. Similarly, the negative pressure tubing will collect the "bone dust" to a separate compartment in the handle, minimizing the health hazard especially in suspected prion diseases. It is also suggested to replace the metal handle with an electrocution resistant material. A smaller version of an electric saw similar to a "can opener" could be devised for finer dissections of the base of the skull (for example, the removal of the pituitary gland or the middle ear) and other skeletal structures elsewhere on the body.

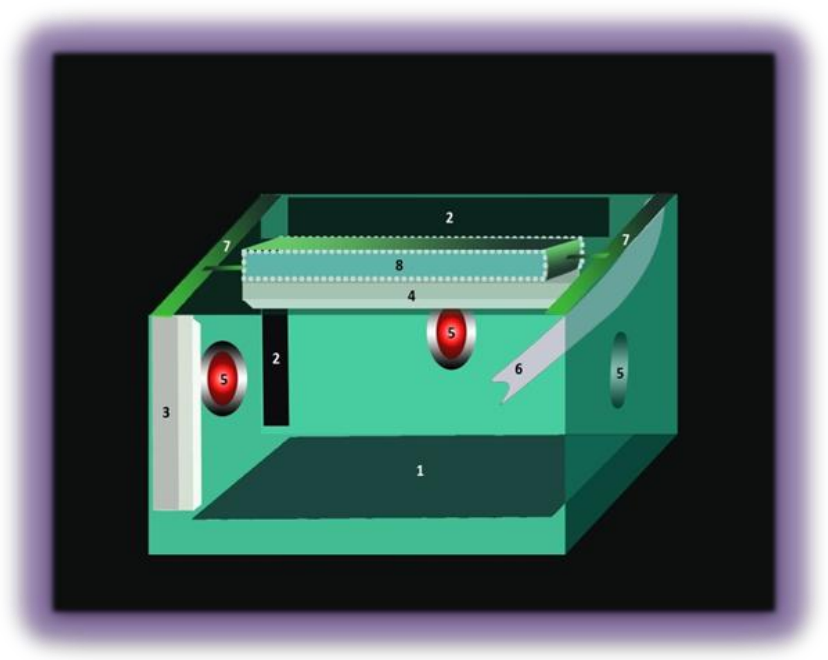

Figure 3: Multi Purpose Brain Dissector

The novel equipment where much sophistication is achieved by incorporation of modern technology would be the multipurpose brain dissector. This equipment is designed in such a way that a human brain, once taken out from the cranial cavity, would be scanned, photographed, dissected (with or without cerebral angiography) and necessary tissue samples taken. This is planned to minimize the manual usage of the conventional brain knife and to overcome some drawbacks in standard dissection. This equipment is operated by a computer program 
and necessary instructions would be provided through a computer to select the required function and to operate the robotic arm. The computer also provides facilities for image archiving simultaneous with dissection of each slice or anatomical part of the brain.

\section{CONCLUSION}

The combined efforts of the forensic pathologists, medical equipment manufacturers and interested parties from other relevant specialties with technological expertise in developing newer instruments would take the dissection techniques to great heights turning the routine autopsy procedure in to a pleasant and more fertile experience.

\section{ACKNOWLEDGEMENT}

Our thanks go to Dr. M.I. Jayaweera and Dr. P.N. Managoda of the Faculty of Medicine, Ragama for their secretarial assistance.

\section{LIMITATIONS}

The authors clearly wish to indicate that the above mentioned instruments are purely conceptualized ideas derived from already existing instruments. The ideas were never tested to see the feasibility of production. However, the scientific thinking would open doors for future expanded ideas and production of user friendly instruments.

\section{REFERENCE}

1. Forensic Medicine from old problems to new challenges: 2011. Chap 14. Duarte Nuno Vieira.

2. www.mopec.com [last accessed on 5.2.2015]

3. www.meder.com [last accessed on 5.2.2015]

4. Jacopo A, Natalie M, Hauke B, Paul M, Colleen S et el. Postmortem examination of patient H.M.'s brain based on histological sectioning and digital 3D reconstruction. Nature Communications 5, Article number: 3122. doi:10.1038/ncomms 4122. 